\title{
Incidental Findings on Liver Autopsy with Specific Emphasis on Hepatitis B
}

\author{
P Jaya Ganesh ${ }^{1}$, R Vimal Chander ${ }^{1 *}$ and J Mahendran ${ }^{2}$
}

${ }^{1}$ Department of Pathology, Saveetha Medical College and Hospital, Thandalam, Chennai, India.

${ }^{2}$ Department of Forensic Medicine, Saveetha Medical College and Hospital, Thandalam, Chennai India

\section{ABSTRACT}

Background: Silent liver disease is a major cause of morbidity and mortality in the population due to its silent progression to end stage liver disease without significant symptoms. These have to be detected at an earlier stage to reduce the morbidity. This study is aims at correlating the gross and microscopic morphology of 100 liver specimens obtained by autopsy and to correlate with the clinical parameters.

Methods: 100 liver specimens obtained by the autopsy of 100 patients, who died of causes like road traffic accidents, poisoning etc. with no medical history during the period from 2007 to 2012, were taken for the study. Histological samples of liver specimens were stained with the routine heamatoxylin and eosin staining, along with special stains like PAS, Reticulin, Perl's Prussian blue and Orcein staining.

Result: The most common pathology was steatosis (25\%) following normal histology (49\%), which was followed by chronic venous congestion (12\%), chronic hepatitis (6\%), cirrhosis (4\%), liver cell dysplasia (2\%) and metastases (2\%). These findings have been correlated with age, sex and special staining. Orcein staining identified ground-glass hepatocytes with detection of Hepatitis B surface antigen in a background of chronic hepatitis and cirrhosis, indication the most probable etiology of Hepatitis B.

Conclusion: This study reiterates the unexpected higher prevalence of NASH among the younger generation and the importance of clinical autopsy in understanding the magnitude of clinically silent liver lesions, mainly Hepatitis B.

Keywords: Hepatitis, Cirrhosis, Orcein Stain, Steatohepatitis, Liver Autopsy.

\section{Introduction}

Silent liver disorders, similar to disease processes in other organs of the body may be symptomatic or may not sometimes produce evident phenotypic features to aid in early diagnosis and treatment. The disease process may progress and then later produce symptoms that may be brought to the purview of the clinician. Though early diagnosis has been made possible with the advent of latest technological and radiological advances, a major portion of disorders remain underdiagnosed which can even progress silently to death. Thorough postmortem examination identifies the cause of death or validates the clinical diagnosis that helps in unleashing the underlying disorders. Pathological processes like fatty liver disease, alcoholic and non-alcoholic fatty liver disease, chronic hepatitis and carriers of the same, etc. produce an indolent course which fails detection through the regularly applied tests until the process becomes more severe. With the increasing prevalence of Hepatitis B in the world, failure to diagnose it in the absence of symptoms increases the chain of transmission thereby producing a vicious cycle. Through this study we would like to study the presence of various diseases in the absence of clinical history by examining the gross and microscopic morphologies of liver specimens correlated with their age and sex prevalence obtained from the autopsy of 100 patients. Also, to identify the pathology of viral hepatitis, especially hepatitis B using the special technique of Orcein staining and to associate its clinical implication in undiagnosed hepatitis B cases which pose a threat to the community.

\section{Materials and Methods}

The material for study, which included liver specimens, was obtained from the autopsy of 100 patients during the period from 2007 to 2012 . The cases were of road/railway accidents, poisonings, etc with no known history of liver disease. The specimens were weighed and examined for gross abnormalities and fixed with $10 \%$ formalin, followed by taking small bits of tissue, 2 each from both the lobes of the liver. Histological samples were stained with the routine heamatoxylin and eosin staining, along with special stains like PAS, Reticulin, Perls Prussian blue and Orcein staining.

Orcein staining is done by preparing Shikatas orcein solution is prepared by dissolving $1 \mathrm{~g}$ of orcein powder in $100 \mathrm{ml}$ of $70 \%$ alcohol. $1 \mathrm{ml}$ of concentrated nitric acid is added to it and kept aside at room temperature for 24 hours. The tissue is then deparaffinized and hydrated with distilled 
water. It is oxidized in freshly prepared acidified potassium permanganate for 5 minutes followed by being bleached in $2 \%$ aqueous solution of oxalic acid for 1 minute or until the sections turn white. The tissues are washed well with water and rinsed briefly with $70 \%$ alcohol. It is now placed in modified Shikatas solution for 10 minutes. Excess stain is removed by placing in $70 \%$ alcohol. The tissues are then studied under the microscope to look for positively stained ground glass hepatocytes.

The samples were studied and analyzed according to the criteria as follows:

Steatosis: presence of fatty change; graded as $1+$ to $4+$ depending on the percentage of cells containing fat.

Steatohepatitis: presence of pericellular fibrosis, portal and acinar inflammation, ballooning degeneration, hepatocyte necrosis associated with fatty change. ${ }^{[1]}$

Chronic venous congestion: presence of sinusoidal dilatation, congestion and presence or absence of centrilobular necrosis.

Chronic hepatitis: interface hepatitis, portal inflammation and spotty necrosis.

Cirrhosis: presence of regenerative nodules and fibrosis.

Liver cell dysplasia: cellular enlargement, nuclear pleomorphism, multinucleation with normal nuclear: cytoplasmic ratio.

Ground glass hepatocytes identified by Orcein staining are graded as Type I- marginal GGH (positive staining of cytoplasmic periphery), Type II- diffuse GGH (diffuse staining), Type III- Globular GGH (globular staining of hepatocytes), Type IV- spotty drop like positive structures, Type V- stained GGH with fatty changes. ${ }^{[2]}$

Each of the above said morphological features were analyzed with respect to various parameters such age, sex, weight of the liver, special stains, etc.

\section{Result}

This study dealt with the morphological and histological examination of liver biopsy specimens obtained through the autopsy of 100 patients, who died of road/railway accidents, poisoning, etc., in which no history of any medical ailment was available. The liver tissue was obtained, sectioned, stained and observed under the microscope following the gross examination of the same.

Out of the 100 cases studied, 49 cases (49\%) showed normal histology [Figure 1]. Fatty change was the most commonly seen morphology in $25 \%$ of cases among which
$8 \%$ were observed to be associated with hepatitis [Figure 2]. Chronic venous congestion was seen in $12 \%$ of cases, which was the second most common. This was followed by chronic hepatitis in $6 \%$, and $4 \%$ with cirrhosis and $2 \%$ each of liver cell dysplasia and metastases. Each of these histological findings were correlated with respect to age, sex and findings on using special stains. This was done by categorizing the cases into 7 groups based on the age, in intervals of 10 years like Group A- 21 to 30 years, Group B-31 to 40 years and so on till Group F- 71 to 80 years.

The finding of steatosis was the most predominantly observed histology in $17 \%$ of cases of which, $88.2 \%$ were males and $11.8 \%$ females. Its incidence was maximum in the age group of $31-40$ years $(41.1 \%)$ followed by $31.3 \%$ in 51-60 years. It was not very evident in cases aged older than 60 years.

In $8 \%$ of the cases with steatohepatitis, $62.5 \%$ were males and the rest, females. Equal incidence was seen in the ages between 31-40 years and 41-50 years, $37.5 \%$ each. No cases were noted with this change in the ages between 21 30 years and $71-80$ years.

Chronic venous congestion was seen in $12 \%$ of cases, of which $83.3 \%$ were males and $16.7 \%$ females. $41.7 \%$ of these cases were seen in the age group of 41-50 and least observed between 61-80 years and not seen in cases aged between 21-30 years.

Among the $4 \%$ of cases with cirrhosis, all the 4 were males $(100 \%)$ with 3 cases $(75 \%)$ aged between $61-70$ years and 1 case $(25 \%)$ between $41-50$ years [Figure 3]. Grossly, two were of micronodular variety, and one each of macronodular and mixed types.

Chronic hepatitis was noted in $6 \%$ of cases among which, the male:female ratio was equal. The major bulk was seen in the age group of 41-50years (66.6\%) [Figure 4].

Liver cell dysplasia was observed in $2 \%$ of cases of which 1 was a male and the other female. Both were noted in the age range of 71-80 years.

Liver metastases were observed in $2 \%$ of cases of which one was male and the other female in the age groups of 5160 years and $41-50$ years respectively.

Special stains were applied to the specimens which gave the following results: PAS for estimating the presence of glycogen and Perl's stain for iron to assess the random prevalence of hemochromatosis did not show significant positivity except for Grade 2+ positivity for increased iron stores in one case in the age group of 61-70 years which was seen in association with micronodular cirrhosis, probably implicating alcohol etiology. Orcein staining was 
done [Figure 5], the technique of which was as proposed by Borchard and Gussman. ${ }^{[3]}$ Among which the maximum positivity was observed in the age range of 41-50 years (5 cases). All of these were seen along side normal histologies, steatosis, cirrhosis or chronic hepatitis. One case was seen with chronic hepatitis and one with cirrhosis $(7.14 \%$ each) which implicated a viral etiology in these cases most probably that of hepatitis $\mathrm{B}$. The other cases were seen with steatosis $(42.8 \%)$, cirrhosis $(14.3 \%)$, chronic hepatitis (7.14\%) and with normal histology in $35.7 \%$ of cases [Figure 6]. Among these, 71.4\% were males and the rest females. Of the five cases with normal histology, four showed type I and one type II. Of the 6 cases with steatosis, type I and type II were shown by $50 \%$ each of the cases.

\section{HISTOMORPHOLOGY OF LIVER ON AUTOPSY}

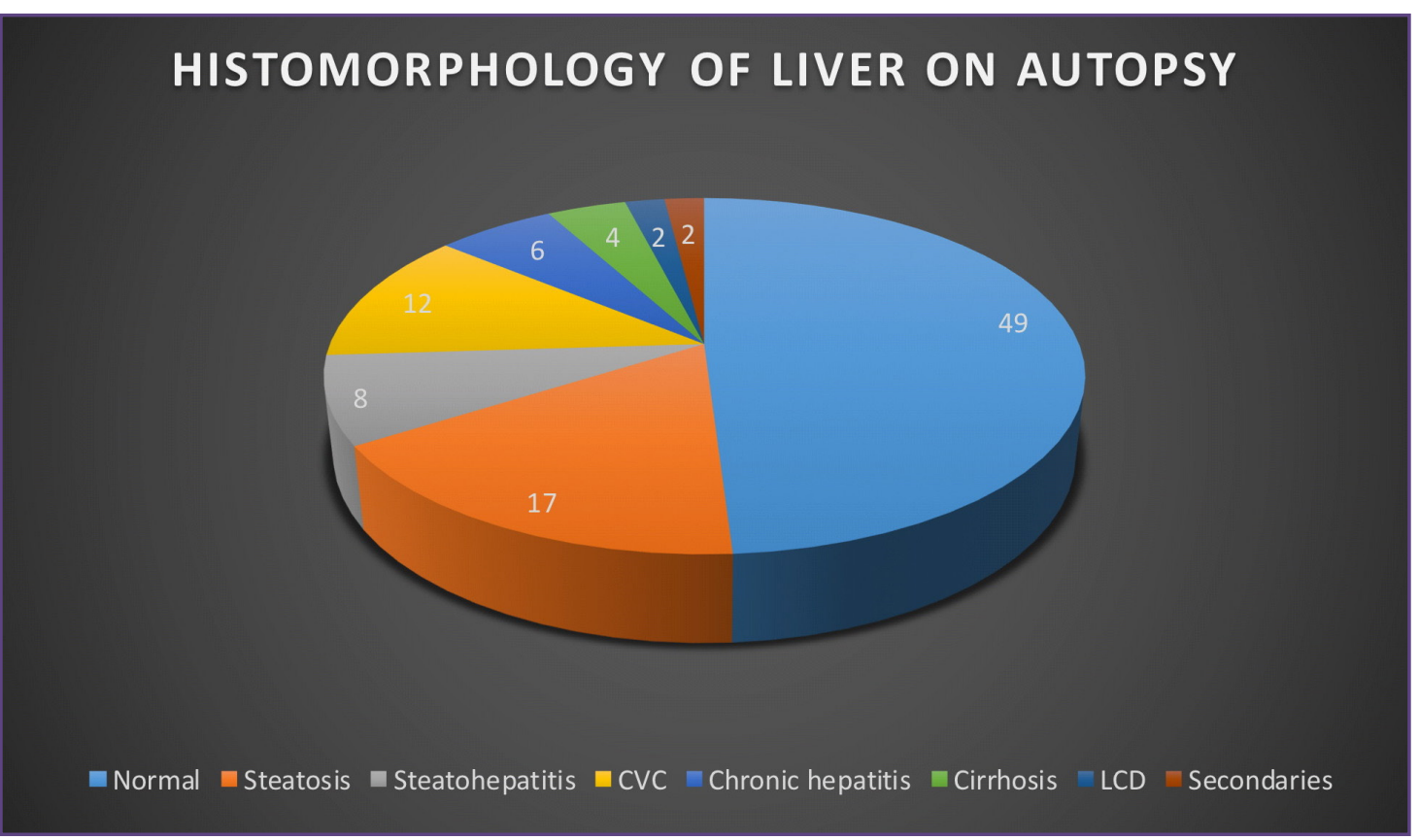

Fig. 1: Histomorphology of liver on autopsy.

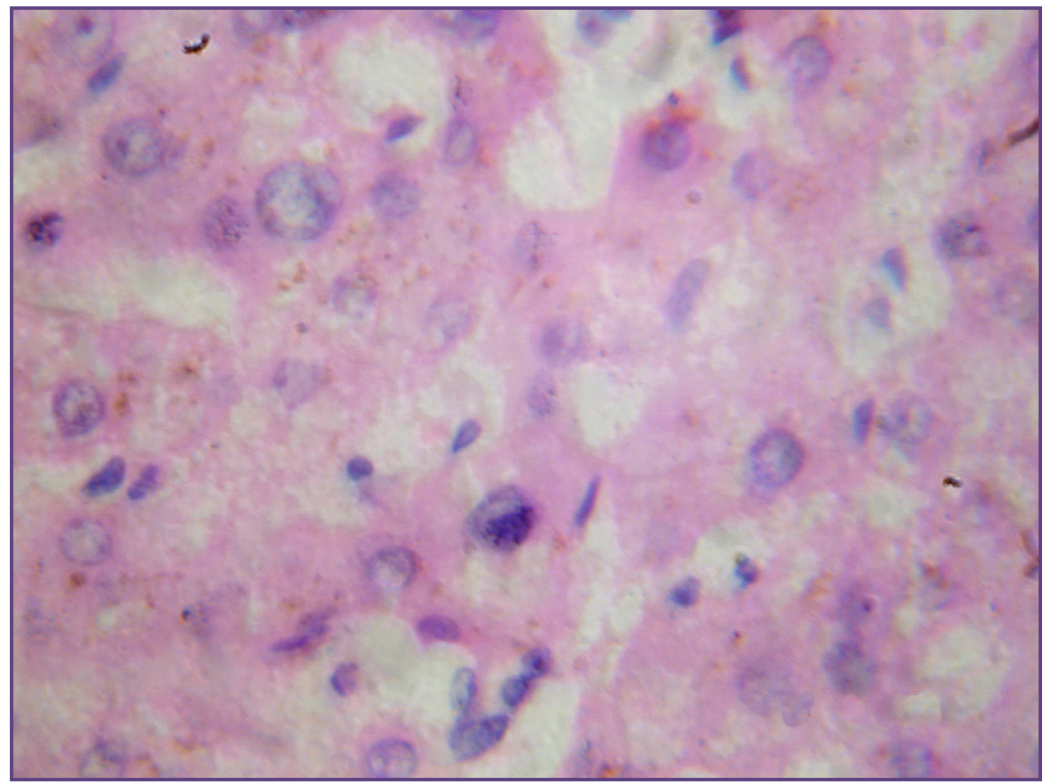

Fig 2: Ground glass hepatocytes H and E x400. 


\title{
CIRRHOSIS-AGE \& SEX DISTRIBUTION
}

\author{
@MALES $\triangle$ FEMALES
}

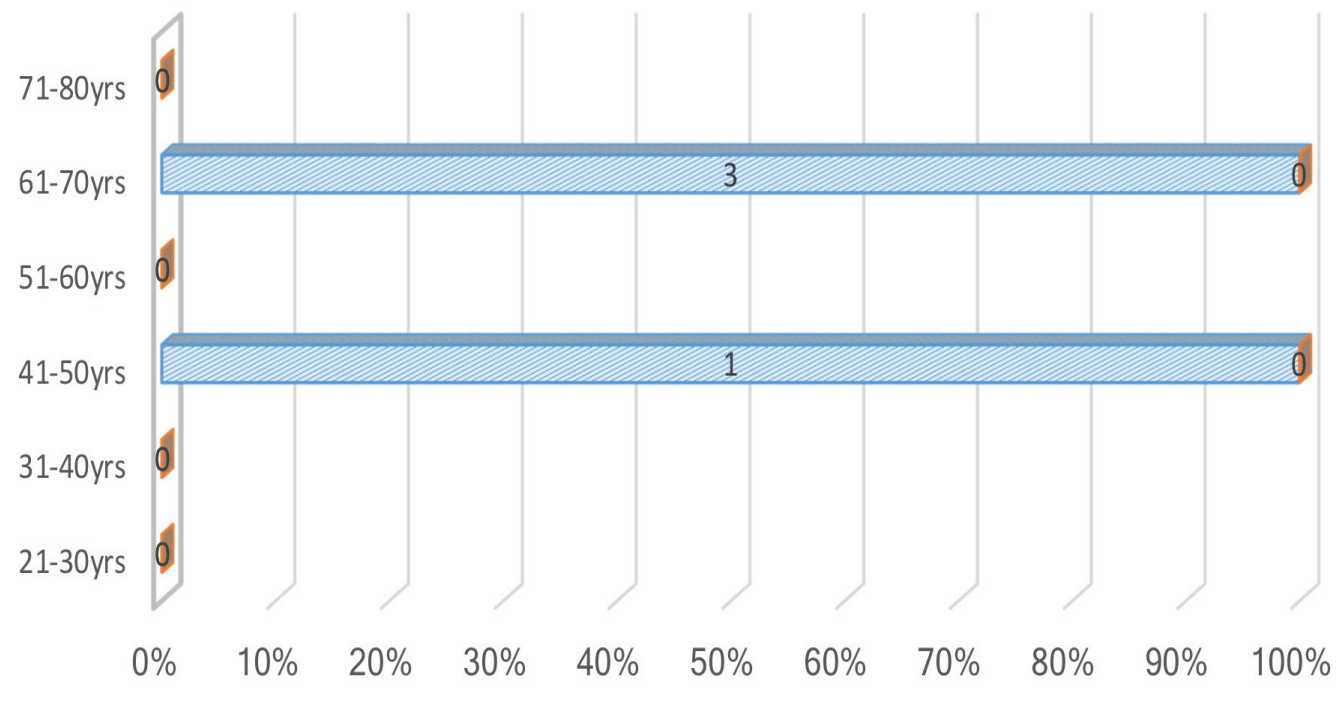

Fig. 3: Cirrhosis - Age and sex distribution.

\section{CHRONIC HEPATITIS-AGE \& SEX DISTRIBUTION}

\section{MMALES MFEMALES}

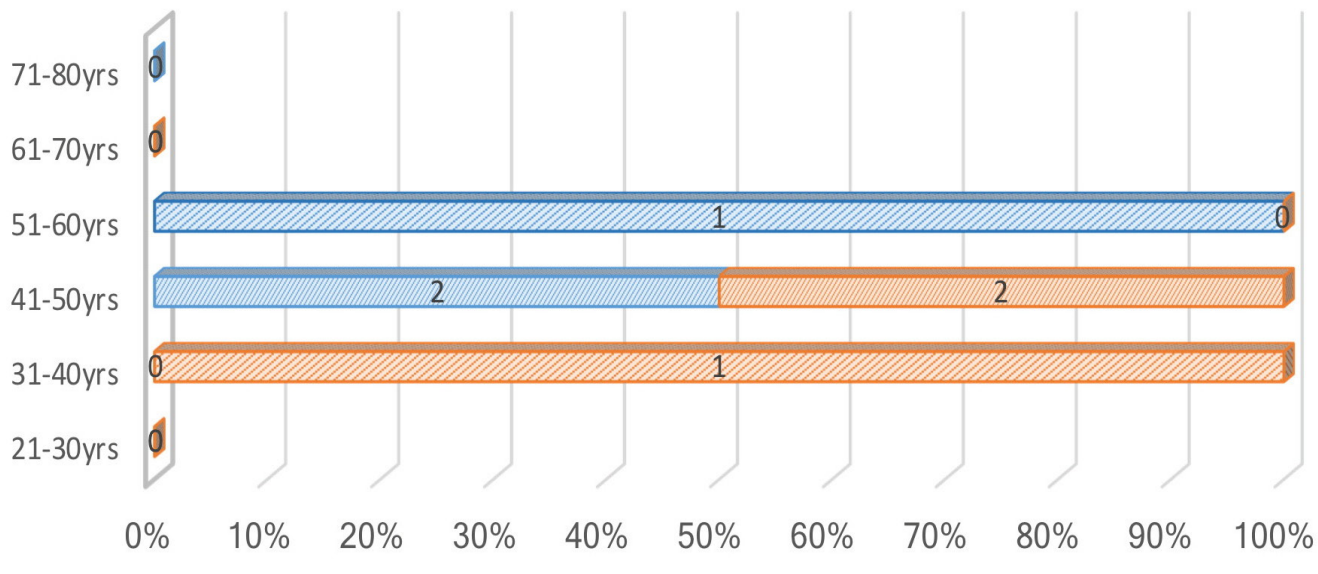

Fig. 4: Chronic hepatitis - Age and sex distribution. 


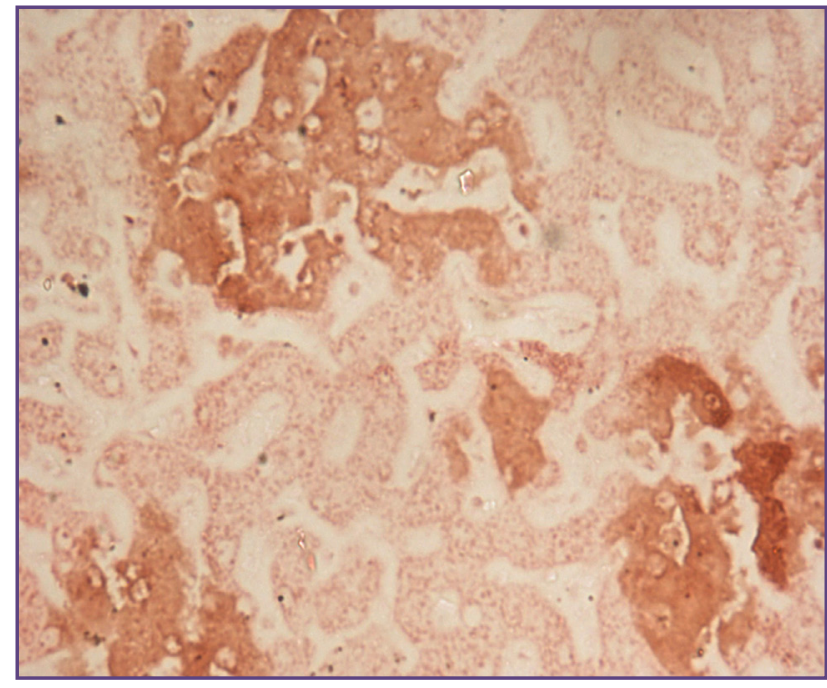

Fig. 5: Positive staining Ground glass hepatocytes - Orcein stain $H$ and $E \times 100$.

\section{Discussion}

Postmortem examination dates back to nearly third century $\mathrm{BC}$ and is being performed till date for various reasons. Though it has been performed for thousands of years, it was during the eighteenth century that it came to be recognized as being fundamental to medical practice. It is one of the best tools to validate clinical diagnosis. ${ }^{[3]}$ Gall in his writeups in the years 1912, 1937,1960 and 1968 has mentioned that "when autopsy records were used to measure the range of clinical error, there was approximately an identical percentage of diagnostic error". Though the reasons for this finding are many, it remains undoubted that clinical diagnosis needs surveillance, the best tool being autopsy. ${ }^{[4]}$ Later, Bauer and Robbins have also suggested that there exists a discrepancy between the clinical diagnosis and anatomical findings, all of which cumulatively suggest the indispensable nature of autopsy to clarify the clinical diagnosis to help in better disease detection and hence in treatment of the cases that follow. ${ }^{[5]}$

Liver is the site of many diseases, many of which become symptomatic, while some are diagnosed only during autopsy. Silent liver diseases, which are becoming an emerging threat to health have to be studied in detail to identify them earlier and provide appropriate treatment. The exact incidence or prevalence of these diseases in unknown because most of these need an invasive investigation in the form of liver biopsy, which is usually not done on a routine basis. In the USA, Steatosis is identified to be the major silent liver disease, while non-alcoholic steatohepatitis constitutes a major minority, whose incidence is increasing. A meta analytic study to estimate the prevalence of NASH showed that the global prevalence is around $25.24 \%{ }^{[6]}$

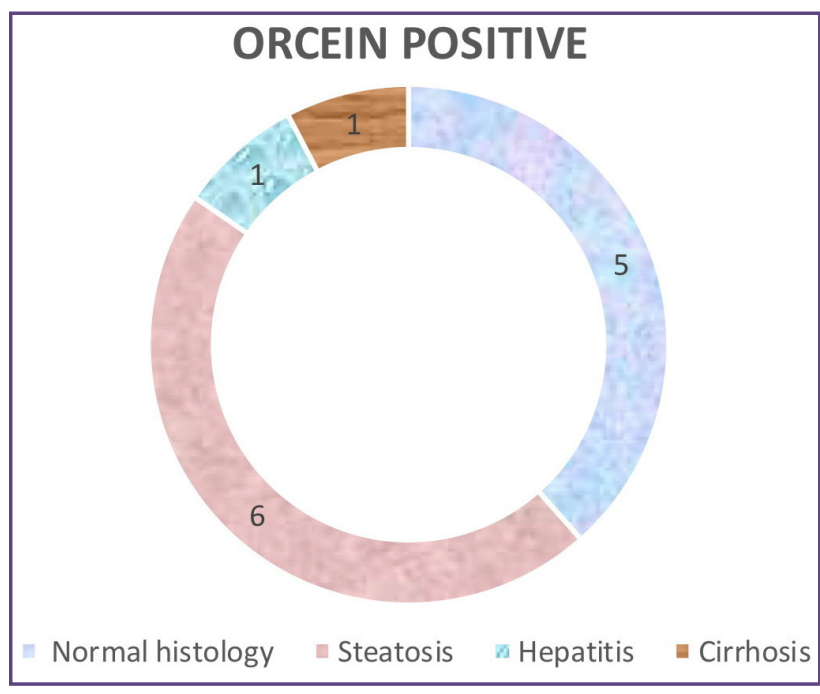

Fig. 6: Orcein positivity in relation with the histopathology.

The term NASH has been coined by Ludwig et al, with the disease process ranging from uncomplicated steatosis to steatohepatitis to steatonecrosis to liver cell failure. ${ }^{[1]}$ In India, alcohol related liver disease is the major health problem. Alcoholic liver disease has been reviewed by many and mentioned in great detail by McSween, Maddrey WC, Rabin and Ishak, et al, however the true incidence of alcoholic hepatits is not knowing owing to the late presentation to the clinician, ending up in cirrhosis. The susceptibility of patients to alcohol related liver disease has been studied by Saunders et al and Sorensons et al.

The other common disease being hepatitis, where extensive research has been done. With the increasing prevalence of Hepatitis B worldwide, its diagnosis and detection of carrier states is needed to reduce the transmission. This is done through serum tests or if asymptomatic, usually diagnosed only on autopsy. Cirrhosis, which is on the extreme end of the spectrum of liver disorders due to various etiologies, can be diagnosed usually on biopsy only, which is seldom done. Morphological classification of the cirrhotic liver helps in establishing the etiology.

Further, using special stains aid in more specific detection of the diseases. Perl's stain for the detection of iron stores in the form of both ferritin or hemosiderin. A definitive grading system was established by Searle JW, Ken JFR, et al. A mild increase in the levels of iron has been observed in cases with non-alcoholic steatohepatitis. The detection of glycogen has been made easier with PAS staining, which was reviewed by Sheehan DC and Luna et al. A special stain called Orcein was developed and later modified by Shikata, which was used to stain hepatocytes for the detection of Hepatitis B surface antigen. ${ }^{[2]}$ Positively staining ground 
glass hepatocytes were graded in five grades. ${ }^{[2]}$ In acute viral hepatitis, no ground glass hepatocytes were found but a few positively staining phagocytes were seen. Types I and II are usually seen in carriers and patients with minimal hepatitis B. in cases with chronic hepatitis, type II and III were observed, while type IV was seen in patients with chronic active hepatitis. Kostich ND, Ingham CD et al, used the orcein and modified Shikata's orcein staining to diagnose carriers and chronic hepatitis.

In our study, steatosis which is the most common histological finding which was mostly seen in the 3rd to 5 th decades of life and also more among the males than females. This was in similarity with the study done by M.S.Bal, S.P. Singh et al, which included patients aged more than 40 years, where in steatosis was seen in $53.85 \%$ cases in the 5 th decade falling to $35.9 \%$ in the 6 th decade. ${ }^{[7]}$

In $8 \%$ of the cases with steatohepatitis, maximum incidence was seen in the third and fourth decades and males are more frequently affected while the female proportion of these cases may implicate non alcoholic steato-hepatitis, which is one of the more common liver pathologies in nonalcoholics. In the study of Soutoudehmanesh, Soutoudeh et al, the prevalence was found to be $2.1 \%$ which compared well with our study, though in lower proportions, which may be attributed to the racial and social differences. ${ }^{[8]}$

Among the cases diagnosed with chronic venous congestion, a higher proportion of males was again noticed with more number of them clustered in the 41-50 years age group. Bal et al, reported a higher percentage of cases in the fifth decade, while the study by Ghazala anif, Hannan et al, reported a much lower proportion of $2.7 \%$ of the same. ${ }^{[7,9]}$

Maximum incidence of cirrhosis was noted among males between the age group of 61-70 yrs. this could probably be attributed to alcoholism. Ghazala anif, Hannan et al, showed a similar incidence of around $4.5 \%$, while, Bal et al, reported a much higher rate of $42.85 \%$ in the $41-50$ years age group. However, Sotoudehmanesh, Soutodeh et al, observed only $0.8 \%$ of cases to be of cirrhosis liver. ${ }^{[7,8,9]}$

Chronic hepatitis was more common among the 4th decade with an equal incidence among males and females. Bal et al, and Soutoudehmanesh et al, reported similar incidences of $3 \%$ and $2.6 \%$ respectively while, Ghazala anif, Hannan et al, reported chronic hepatitis in $12.7 \%$ of cases. ${ }^{[7,8,9]}$

Liver cell dysplasia was observed in only $2 \%$ of cases and this finding was not reported in most of the earlier studies. However Anthony et al reported its presence of $1 \%$ in normal appearing livers. A few cases were positively associated with Hepatitis B along with its usual correlation with hepatocellular carcinoma. Liver is a common site for metastases, which has been observed in $2 \%$ of cases in our study. Borja ER, Hori et al, reported 25 cases of carcinomatous cirrhosis with secondaries from breast carcinoma. ${ }^{[10]}$ The diagnosis of the primary from liver examination can be done by immunohistochemical methods and detection by histopathology is not possible.

Orcein stain, which is specific for Hepatitis B surface antigen in the ground glass hepatocytes, was done to assess the random prevalence of Hepatitis B surface antigen with a sensitivity of around $80 \%$ in cases with minimal changes, chronic hepatitis and cirrhosis. It stains the viral coat better than the core antigen. It therefore cannot be used as a test for primary detection of Hepatitis B, but nevertheless, its use in detection of carriers has been proven to be effective. Shikata's stain also shows positivity with elastic fibrous tissue, thereby contributing to its findings in those lesions apart from those seen in Hepatitis B. It also stains positively with copper inclusions, which needs to be excluded if significant. Cases of chronic venous congestion showed type I positivity. In the patients with cirrhosis, one showed type I and the other type III, whereas in cases of chronic hepatitis, type III was observed. Types I and II indicate carrier state and minimal hepatitis, whereas, types II and III are observed in chronic hepatitis. In a study done by N C Nayak and R. Sachdeva, The effectiveness of orcein staining has been compared with immunoperoxidase methods and was found been to be as effective as the latter and less time consuming, though the sensitivity is more. ${ }^{[1]}$ This shows that orcein staining is a good tool to identify Hepatitis B surface antigen, though few false negative or positive cases may occur.

\section{Conclusion}

This study has helped in identifying hepatitis B in the autopsy specimens through special staining, which could have had far reaching consequences both clinically and socially in the form of spread of the disease from asymptomatic cases or carriers. This has also been felt to be a major health hazard to the health care professionals, which can be reduced by its earlier detection. Steatosis, though a normal finding in many aged people, has been seen as early as 22 years of age. In our population of study, chronic venous congestion, chronic hepatitis, steatohepatitis and cirrhosis were seen as early as around 40 years of age and clustered more in the 4 th and 5 th decades of life. Steatohepatitis was found among the females which points to the probable possibility of non-alcoholic steatohepatitis, also implicating the unexpected higher prevalence of NASH among the younger generation and that metabolic syndrome continues to be underdiagnosed among the female population. This study thus reiterates the importance of clinical autopsy in understanding the magnitude of clinically silent liver lesions, mainly Hepatitis B. 


\section{Reference}

1. Brent A, Neuschwander-Tetri et al Non-Alcoholic steatohepatitis. Summary of an AASLD Single topic Conference. J Hepatology 2003;(37):1202 -1219.

2. Mani H, Kleiner DE. Liver Biopsy Findings in Chronic Hepatitis B. Hepatology 2009;49(5):S61-S71.

3. Burton JL. A bite into the history of the autopsy : From ancient roots to modern decay. Forensic Sci Med Pathol 2005 Dec;1(4):277-84.

4. Silas OA, Adoga AA, Manasseh AN, Echejoh GO, Mandong BM, Olu-Silas R. The Role of Necropsy in Diagnostic Dilemmas as Seen in a Tertiary Hospital in North Central Nigeria. Journal of Tropical Medicine 2009 (2009), Article ID 718984. DOI: $10.1155 / 2009 / 718984$

5. Disibio G, French SW. Metastatic patterns of cancers: results from a large autopsy study. Arch Pathol Lab Med. 2008 Jun;132(6):931-9. DOI: 10.1043/1543-2165

6. Younossi ZM, Koenig AB, Abdelatif D, Fazel Y, Henry L, Wymer M Global epidemiology of nonalcoholic fatty liver
disease-Meta-analytic assessment of prevalence, incidence, and outcomes. Hepatology 2016;64(1):73-84.

7. Bal MS, Singh SP, Bodal VK, Oberoi SS, Surinder K Pathological findings in Liver Autopsy. JIA Forensic Medicine 2004;26(2):55-57.

8. Sotoudehmanesh R, Sotoudeh M, et al Silent liver diseases in autopsies from Forensic Medicine of Tehran. Arch Iran Med 2006;9(4):324-28.

9. Ghazala Hanif, Abdul Hannan Nagi Incidental findings in the liver - An Autopsy study. Ann King Edward Med Coll 2001;7(1):58-60.

10. Sass DA, Clark K, Grzybicki D, Rabinovitz M, ShawStiffel TA. Diffuse desmoplastic metastatic breast cancer simulating cirrhosis with severe portal hypertension: a case of "pseudocirrhosis". Dig Dis Sci. 2007;52:749-752.

11. Mukhopadhya A, Ramakrishna B, Richard V, Padankatti R, Eapen CE, Chandy GM. Liver histology and immunohistochemical findings in asymptomatic Indians with incidental detection of hepatitis B virus infection. Ind J Gastroenterol 2006;25:128-131.

*Corresponding author:

Dr. R Vimal Chander, 216, Mahatma Gandhi 2nd Street, Thiruverkadu Cooperative Nagar, Thiruverkadu, Chennai - 600077. India

Phone: +91 9790645729

Email: rvimalchander@gmail.com

Date of Submission : 20.03.2017

Date of Acceptance : 14.06.2017

Financial or other Competing Interests: None.

Date of Publication : 25.10.2017 